\title{
Métodos para la estimación de costos en salud de la EPOC: resultados basales*
}

\author{
Rosario Fernández-Plata, David Martínez-Briseño, $₫$ Cecilia García-Sancho Figueroa, \\ Dalia Cano-Jiménez, Alejandra Ramírez-Venegas, Raúl Sansores-Martínez, Luis Torre-Bouscoulet, \\ Guillermo Casas-Medina, Rogelio Pérez-Padilla
}

Instituto Nacional de Enfermedades Respiratorias Ismael Cosío Villegas, Ciudad de México.

Trabajo recibido: 27-VII-2015; aceptado: 27-X-2015

\begin{abstract}
RESUMEN. Antecedentes: El estudio PLATINO realizado en la Ciudad de México (2003) mostró una prevalencia de EPOC de 7.8\%. México no cuenta con información publicada sobre gastos directos e indirectos en salud que realizan los pacientes con EPOC. Objetivo: Estimar los costos directos e indirectos de pacientes con EPOC en un hospital de tercer nivel en México. Material y métodos: Se entrevistó a pacientes con EPOC en cada consulta ambulatoria durante un año. En la entrevista basal preguntamos por los gastos de bolsillo del año previo al reclutamiento. Asimismo, se registraron los costos para el hospital. En este trabajo analizamos los costos anuales en dólares de 2014 previos al reclutamiento de pacientes ambulatorios con $\geq 12$ meses de diagnóstico estratificando por categorías GOLD. Resultados: Se reclutaron 611 pacientes, de los cuales 484 tuvieron un diagnóstico $\geq 12$ meses. La mediana de los costos directos anuales por parte de los pacientes por GOLD fueron: $\$ 1,116$, leve; $\$ 1,533$, moderado; $\$ 1,825$, grave; $\$ 2,245$, muy grave, que difieren estadísticamente ( $<$ < 0.01). Conclusión: Los costos directos se incrementan con la gravedad de la EPOC y se debe principalmente al gasto en medicamentos, oxígeno y alojamiento. Es importante apegarse a una metodología estricta para que los resultados sean comparables.
\end{abstract}

Palabras clave: EPOC, costos directos, costos indirectos, gasto de bolsillo.

ABSTRACT. Background: The PLATINO study developed in Mexico City (2003) showed a COPD prevalence of $7.8 \%$ in individuals 40 years of age and older. In Mexico, there is no published information concerning direct and indirect health related costs for patients with COPD. Objective: To measure direct and indirect costs of outpatients with COPD at a tertiary-level hospital in Mexico. Material and methods: We recruited outpatients with diagnosis of COPD during one year. At base-line interview we asked about out of pocket health expenses for the last year previous to enrollment. Costs for the hospital were registered as well. For this study, we analyzed costs in 2014 US dollars for the last year previous enrollment in patients with > 12 months of being diagnosed stratified by GOLD categories. Results: We enrolled 611 outpatients with COPD, of whom 483 had a time of diagnosis > 12 months. The medians of annual direct costs to patients by GOLD were: $\$ 1,116$, mild; $\$ 1,533$, moderate; $\$ 1,825$, severe, and $\$ 2,245$, very severe. The differences among the medias of annual direct cost were statically signified ( $p$ < 0.01). Conclusions: Direct out of pocket costs increased with COPD severity and were due to drugs, oxygen, and accommodations mainly. It is important to have a standardized methodology to make the results comparable.

Key word: COPD, direct costs, indirect costs, out-of-pocket expenses.

\section{INTRODUCCIÓN}

La enfermedad pulmonar obstructiva crónica (EPOC) debida primordialmente al tabaquismo se caracteriza funcionalmente por la obstrucción del flujo aéreo, pero

*. Este estudio fue financiado con el apoyo irrestricto de Novartis México (número de acuerdo 001-MX13-CQAB149 y título de protocolo «Estimación de costos de la enfermedad pulmonar obstructiva crónica (EPOC) en un hospital de tercer nivel desde la perspectiva social»: Seguimiento de un año. La compañía no participó en el diseño y realización del estudio, ni en el análisis de los datos y escritura del artículo. está asociada a varias comorbilidades. La Organización Mundial de la Salud estimó que más de tres millones de personas murieron debido a la EPOC en 2012, representando $6 \%$ de las muertes a nivel mundial, de las cuales $90 \%$ ocurrió en países de ingresos bajos y medios. ${ }^{1}$ La EPOC es actualmente la tercera causa de muerte y será la quinta causa de discapacidad para 2030 a nivel internacional. ${ }^{2}$ Los costos en salud de la EPOC son cuantiosos, ${ }^{3}$ pero las estimaciones suelen provenir de países de ingresos altos ${ }^{4-11}$ y se han descrito para países como Estados Unidos, Inglaterra, España, Rumania y Colombia. ${ }^{12-20}$ 


\begin{abstract}
El estudio PLATINO realizado en la Ciudad de México en 2003 mostró una prevalencia de $7.8 \%$ para la EPOC, la cual es considerable para el sistema de salud aunque de las más bajas reportadas. ${ }^{21}$ La mortalidad por la EPOC en México se ha incrementado y en personas mayores de 65 años se situó en el quinto lugar dentro de las 10 primeras causas de mortalidad en $2005 .{ }^{22} \mathrm{La}$ carga financiera se ha descrito sólo desde la perspectiva del sistema de salud en México. ${ }^{23-25}$ Para evaluar de forma adecuada la carga económica de la EPOC es necesario contar con información sobre los costos desde la perspectiva del paciente y de los factores que explican su variabilidad. El objetivo de este estudio fue estimar los costos directos e indirectos de un grupo de pacientes con EPOC durante 12 meses de seguimiento que acudieron para su atención a un hospital de referencia de enfermedades respiratorias en la Ciudad de México, se realizó el seguimiento por un año completo con el propósito de captar las fluctuaciones de los costos debido a la etiología de la EPOC. En este artículo describimos los métodos utilizados en la estimación de costos en salud y la información basal de pacientes ambulatorios con diagnóstico confirmado de más de 12 meses al momento de su inclusión.
\end{abstract}

\section{MATERIAL Y MÉTODOS}

\section{Estudio, población e instrumentos}

Se realizó un estudio de seguimiento del 1 de agosto de 2013 al 31 de julio de 2014 en el Instituto Nacional de Enfermedades Respiratorias «Ismael Cosío Villegas» (INER), el cual es un hospital de referencia para enfermedades respiratorias que atiende principalmente población no asegurada de la Ciudad de México, del área metropolitana y estados colindantes. Se incluyó consecutivamente a los pacientes que aceptaron participar de acuerdo con los siguientes criterios: hombres y mujeres de 40 años de edad o más, con diagnóstico de EPOC confirmado por espirometría. La captación se realizó en la consulta externa en la Clínica de EPOC y en el pabellón cinco, donde se hospitalizan específicamente pacientes con esta enfermedad. La invitación a participar en el estudio se hizo a todos los pacientes que acudieron a los servicios anteriormente mencionados durante el período de seguimiento. Se excluyó a los pacientes con diagnóstico de asma. El proyecto fue aprobado por el Comité de Ciencias, Bioética e Investigación del INER (número de aprobación S01-12), todos los participantes firmaron consentimiento informado.

Se realizó una entrevista en cada consulta médica a la que acudió el paciente en el período de seguimiento. El cuestionario que se aplicó contenía las siguientes secciones: características sociodemográficas, costos previos al diagnóstico de EPOC, costos directos, costos indirectos, exacerbaciones, calidad de vida (EQ-5D), comorbilidades y gasto familiar en el hogar.

En la entrevista basal, la información para las secciones de costos directos, costos indirectos y exacerbaciones se preguntó acerca del año previo a la inclusión del estudio, mientras que en las entrevistas de seguimiento sólo se preguntó sobre el período entre la consulta médica previa (entrevista anterior) y la consulta médica actual. Las secciones de calidad de vida y comorbilidades se aplicaron en cada entrevista.

\section{Medición de las características sociodemográficas}

En el Servicio de Trabajo Social los pacientes fueron entrevistados para obtener sus características sociodemográficas mediante un cuestionario estructurado y asignarles un nivel socioeconómico. Éste se determina por medio de un índice ponderado que toma en cuenta el ingreso por dependiente (factor con el mayor peso), las características de la vivienda (material de construcción, servicios públicos con los que se cuenta, si la ubicación de la vivienda es urbana, suburbana o rural), si se es propietario o arrenda la casa, y la presencia de otras enfermedades en otros miembros de la familia. El nivel socioeconómico cuenta con diez categorías (0-9). Los pacientes con el nivel socioeconómico más bajo (nivel 0) no pagan ninguna cuota por los servicios médicos recibidos. A medida que se incrementa el nivel, aumenta la cuota que debe pagar el paciente. Los pacientes con nivel 6 pagan el costo real del servicio para el INER. Finalmente, los pacientes clasificados entre los niveles 7-9, pagan el costo real del servicio más una cantidad adicional que representa una ganancia para el instituto.

\section{Costos previos al diagnóstico de EPOC}

Esta sección se dividió en dos partes: 1) referente a los costos médicos realizados en el INER y 2) costos en otras instituciones de salud. Al ser un hospital de referencia de tercer nivel, los pacientes usualmente acuden a otras instituciones de salud previo a ser atendidos en el INER y algunos de ellos lo hacen sin tener aún un diagnóstico preciso.

\section{Perspectiva}

La perspectiva es el punto de vista desde el cual los costos o beneficios son registrados y evaluados, las perspectivas posibles son la del sistema de salud (hospital o proveedor de servicios de salud) o la pers- 
pectiva del paciente. En el presente trabajo se aborda la perspectiva del paciente.

\section{Costos directos}

Comprenden el consumo de los recursos resultantes de un tratamiento o terapia directamente atribuibles a una enfermedad, los costos directos se dividieron en dos grupos: médicos y no médicos. Los costos médicos surgen directamente del tratamiento, mientras que los no médicos resultan de las consecuencias de la enfermedad o del tratamiento. Los costos médicos incluyeron las consultas médicas, pruebas de laboratorio, las recargas del tanque de oxígeno y el uso de equipo para desplazarse. Los expedientes médicos fueron revisados para confirmar las pruebas de laboratorio realizadas a los pacientes. Los precios unitarios para las consultas médicas y las pruebas de laboratorio se obtuvieron de la Unidad de Costos del INER, el equipo para desplazarse incluyó el uso de muletas, bastón, silla de ruedas y andaderas; los pacientes dieron los costos unitarios de los diferentes implementos y únicamente se incluyó este costo cuando se adquirían por los problemas de la EPOC. Los costos de oxígeno incluyeron la compra de tanque o concentrador, mantenimiento, recargas y el consumo de luz, el costo de compra del tanque y concentrador se anualizaron usando una tasa de interés de $4 \%$ y una vida útil de cinco años. Para estimar el costo de luz se preguntó por el cargo antes y después de haber comprado el concentrador de oxígeno; la diferencia se utilizó como el costo de la luz.

Para los costos no médicos se incluyeron los costos por transporte, comida y alojamiento, se preguntó por el costo promedio invertido en estos tres aspectos en cada visita médica. Asimismo, se preguntó por el número de días de alojamiento en casa de familiares o amigos para acudir a sus citas médicas al INER. Cuando los pacientes no reportaban gasto por alojamiento, se contabilizó el número de días que pasaron en casa de un familiar y se asignó un costo de US \$15.16 por día para estimar el gasto anual en alojamiento, considerando los precios de mercado. Lo anterior se realizó para estimar los costos en los que incurriría el paciente si no tuviera en dónde quedarse al venir a sus consultas médicas.

\section{Costos indirectos}

Se definen como la pérdida de productividad o de salario por la ausencia en el lugar de trabajo debido a una enfermedad. Los costos indirectos se estimaron usando el método del capital humano, el cual consiste en la asignación de un salario de acuerdo con la actividad económica que reportaba el paciente, ${ }^{26}$ para ello se utilizó la tabla de salarios mínimos publicada por la "Comisión Nacional de Salarios Mínimos» (CONASAMI) para asignar el salario por día de los pacientes dedicados a algún oficio. ${ }^{27}$ Por otro lado, utilizamos el promedio del ingreso per capita de 2013 y 2014 publicado por el Banco Mundial para estimar el salario de un día de los pacientes con estudios de licenciatura o más (i.e. US \$27.56). ${ }^{28}$ Para los pacientes jubilados o pensionados estimamos su ingreso diario a partir del ingreso anual para trabajadores retirados en México publicado por la «Organización para la Cooperación y el Desarrollo Económicos» (OCDE) en 2013 (i.e. US \$11.78). ${ }^{29}$ Usamos los precios de mercado para estimar el salario diario de las trabajadoras domésticas (US $\$ 13.95$ por día). Asimismo, a los pacientes con alguna actividad laboral se les cuestionó sobre los días de trabajo y de esparcimiento perdidos, para lo cual se asignó el salario diario de las trabajadoras domésticas para los costos de los días de esparcimiento perdidos. Una vez calculados los salarios diarios, se multiplicó el número de días de trabajo y esparcimiento perdidos por su correspondiente salario diario asignado para estimar los costos indirectos de los pacientes, de forma similar calculamos los costos para los acompañantes de los pacientes, todos los costos se expresaron en dólares de Estados Unidos (US) de 2014.

\section{Exacerbaciones}

En esta sección se indagó por el número de exacerbaciones de la EPOC, los costos directos médicos y los indirectos. También se preguntó si tuvo que ser hospitalizado el paciente debido a las exacerbaciones y si la atención hospitalaria la recibió en el INER o en otra institución pública o privada.

\section{Calidad de vida}

La calidad de vida relacionada con la salud se midió en cada visita médica por medio del instrumento EuroQol 5 (EQ-5D), donde el propio individuo valora su estado de salud en niveles de gravedad utilizando un sistema descriptivo que incluye cinco dimensiones: 1) movilidad, 2) cuidado personal, 3) actividades cotidianas, 4) dolor o malestar y 5) ansiedad y depresión. Asimismo, este instrumento cuenta con una escala analógica y permite obtener un índice de valores sociales que se obtiene para cada estado de salud. ${ }^{30}$

\section{Comorbilidades}

En esta sección se preguntó a los pacientes si un médico les había dicho que tenían alguna de las siguientes 
comorbilidades o condición de salud: diabetes mellitus, presión arterial alta, asma, enfermedad del corazón, enfisema, gastritis, úlcera, reflujo, obesidad, tuberculosis, depresión u otra enfermedad o condición de salud. Se preguntó por los medicamentos para tratar cada una de las comorbilidades y el costo total de los medicamentos usados.

\section{Gasto familiar en el hogar}

En esta sección se indagó por el gasto realizado por la familia en alimentos y bebidas para el consumo dentro y fuera del hogar, bebidas alcohólicas, cigarros, transporte local, artículos de limpieza, vestido, calzado, educación distracción, renta, gas, electricidad, agua, luz, teléfono, cuidados en salud, muebles, mantenimiento para la vivienda, vehículos y sus refacciones. Esta información se obtuvo únicamente en la entrevista basal.

\section{Costos de hospitalización}

Mediante la Unidad de Costos del INER se obtuvieron los días de estancia hospitalaria, la cantidad de los insumos médicos y los costos en los que incurrió el paciente durante su internamiento de acuerdo con su nivel socioeconómico. La información anterior se obtiene realizando un prorrateo (reparto de una cantidad en forma proporcional) de todos los insumos que entran en cada almacén (farmacia, víveres y general), gastos de servicios generales, activo fijo (ponderación y utilización de promedios para determinar el gasto de la depreciación por centro de gestión). Los costos de medicamentos y materiales de curación en procedimientos quirúrgicos y de hospitalización se asignan de acuerdo con los porcentajes de pago del nivel socioeconómico del paciente, aunque se cuenta también con los costos reales sin subsidio.

Dependiendo de la gravedad del paciente se realizó lo siguiente: 1) si el paciente estaba inconsciente o intubado, se captaba la información del expediente y se entrevistaba al familiar para obtener la información sobre gastos que se generan por cuidado del familiar (alimentos, transporte, alojamiento, días de trabajo perdidos y si otros familiares o amigos apoyaban en el cuidado; en ocasiones los familiares, además tenían que gastar en medicamentos que no podía proporcionar el hospital), 2) si el paciente estaba consciente y con ánimo, se le informaba brevemente del proyecto en un formato estandarizado de una página (considerando la condición de estos pacientes muy pocos contaban con información de los gastos causados por su internamiento; por lo tanto, sólo se preguntaba por días de trabajo perdido). Posteriormente, en la consulta externa se captaba nuevamente al paciente, en donde se le aplicaba el cuestionario completo.

\section{Análisis estadístico}

Se estimaron estadísticas descriptivas de las variables sociodemográficas, la clasificación de gravedad (estadío GOLD), causa atribuible y los días de trabajo perdidos por el paciente y sus acompañantes del total de pacientes incluidos en el estudio. En el presente análisis utilizamos únicamente la información basal (datos reportados del año previos al reclutamiento) de pacientes con 12 meses o más de haber sido diagnosticados con EPOC. Mediante la prueba de Kruskal-Wallis comparamos las medianas de los costos directos e indirectos en salud estratificando por gravedad, usamos los criterios de la Iniciativa Global para la Enfermedad Pulmonar Obstructiva Crónica (GOLD). Los datos fueron analizados con STATA SE ver.12 (STATA Corporation, College Station, TX, USA 2011).

\section{RESULTADOS}

\section{Características sociodemográficas}

Se invitó a participar en el estudio a 655 pacientes, de los cuales 611 (93.3\%) aceptaron participar en el estudio. Del total de pacientes 324 (53\%) fueron hombres, el promedio ( \pm desviación estándar [DE]) de edad fue de 72.1 ( \pm 9.2$)$ años, 377 (61.7\%) tenía como lugar de residencia la Ciudad de México. 356 (58.3\%) pacientes tuvieron $\leq 6$ años de escolaridad formal. El porcentaje de pacientes que contaban con seguridad social fue de $61.7 \%$; los pacientes con al menos un año de diagnóstico fueron 484 (79.2\%). La frecuencia (\%) por categoría de gravedad (GOLD) fueron las siguientes: leve, 40 (6.5); moderado, 240 (39.3); grave, 153 (25.0) y muy grave, 178 (29.1) (tabla 1).

\section{Costos directos}

De los costos directos médicos, los medicamentos y el oxígeno fueron los principales rubros a los que se destinó el gasto del bolsillo de los pacientes con EPOC, mientras que para el oxígeno no se observaron diferencias significativas en los costos entre las categorías de gravedad (GOLD), los costos en medicamentos mostraron una asociación positiva con la gravedad de la EPOC y las diferencias fueron estadísticamente significativas $(p<0.01)$. El costo por alojamiento fue el rubro más cuantioso de los costos médicos indirectos; sin embargo, no hubo una diferencia estadísticamente 
Tabla 1. Características sociodemográficas de pacientes $(n=611)$.

\begin{tabular}{|c|c|}
\hline Variable & $\mathrm{n}(\%)$ \\
\hline Hombre & $324(53.0)$ \\
\hline Edad (años) [media(DE*)] & $72.1(9.2)$ \\
\hline Lugar de residencia (Ciudad de México) & $377(61.7)$ \\
\hline \multicolumn{2}{|l|}{ Años de educación } \\
\hline$\leq 6$ & $356(58.3)$ \\
\hline $7-12$ & $122(20.0)$ \\
\hline$>12$ & $133(21.8)$ \\
\hline Seguridad social ${ }^{\ddagger}$ & $377(61.7)$ \\
\hline \multicolumn{2}{|l|}{ Nivel socioeconómico (otorgado por el INER) } \\
\hline 0 & $90(14.7)$ \\
\hline 1 & $145(23.7)$ \\
\hline 2 & $163(26.7)$ \\
\hline 3 & $94(15.4)$ \\
\hline 4 & $100(16.4)$ \\
\hline 5 & $19(3.1)$ \\
\hline \multicolumn{2}{|l|}{ Situación laboral } \\
\hline Retirado & $257(42.1)$ \\
\hline Trabajo doméstico & $213(34.9)$ \\
\hline Asalariado & $141(23.1)$ \\
\hline \multicolumn{2}{|l|}{ Gravedad (GOLD) } \\
\hline Leve (I) & $40(6.5)$ \\
\hline Moderado (II) & $240(39.3)$ \\
\hline Grave (III) & $153(25.0)$ \\
\hline Muy grave (IV) & $178(29.1)$ \\
\hline $\begin{array}{l}\text { Años con el diagnóstico de EPOC } \\
\left.\text { [media }\left(\mathrm{DE}^{\S}\right)\right]\end{array}$ & $5.2(4.7)$ \\
\hline \multicolumn{2}{|l|}{ Causas de la EPOC§ } \\
\hline Tabaquismo & $362(59.2)$ \\
\hline Biomasa & $174(28.5)$ \\
\hline Tabaco pasivo & $3(0.5)$ \\
\hline Combinación de causas & $72(11.8)$ \\
\hline Consultas médicas [media(DE)] & $2.8(1.4)$ \\
\hline $\begin{array}{l}\text { Días de trabajo perdidos por el paciente } \\
\text { [media(DE)] }\end{array}$ & $5.0(9.3)$ \\
\hline $\begin{array}{l}\text { Días de ocio perdidos por el paciente } \\
\text { [media(DE)] }\end{array}$ & $6.0(9.7)$ \\
\hline $\begin{array}{l}\text { Días de trabajo perdidos por la familia } \\
\text { [media(DE)] }\end{array}$ & $4.9(5.6)$ \\
\hline
\end{tabular}

* Desviación estándar.

₹ Incluye la afiliación a alguna de las siguientes instituciones: Instituto Mexicano del Seguro Social (IMSS), Instituto de Seguridad y Servicios Sociales de los Trabajadores del Estado (ISSSTE), Sistema de Protección Social en Salud (Seguro Popular).

$\S$ La temporalidad de la exposición es en cualquier momento (pasada o actual).

significativa entre las categorías de gravedad. El total de los costos directos mostró una tendencia positiva y estadísticamente significativa $(p<0.01)$ de acuerdo con la clasificación GOLD: leve (I), US $\$ 1,116$; moderado (II), US \$1,533; grave (III), US \$1,825; muy grave, US $\$ 2,245$ (IV) (tabla 2). Asimismo, se observó una mayor dispersión en el total de los costos directos a medida que aumentaba la gravedad de la EPOC (figura 1).

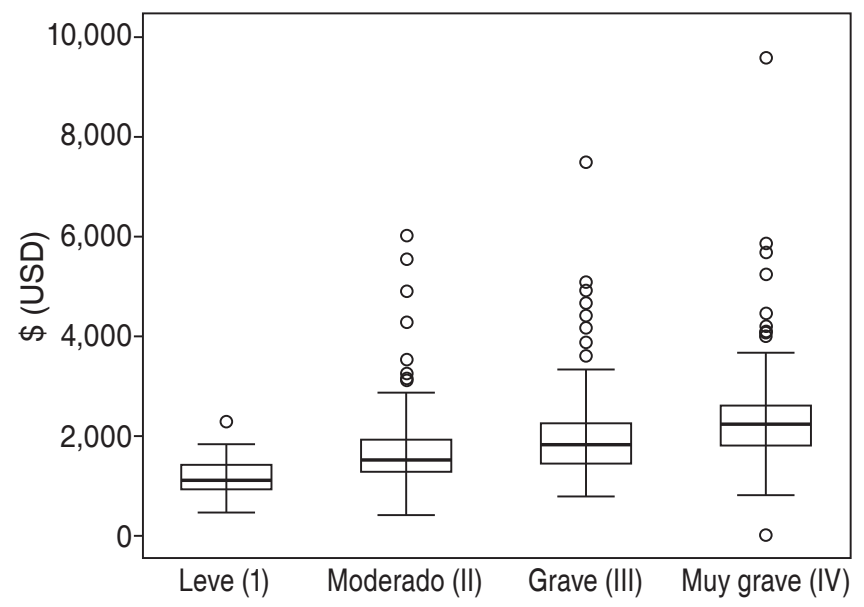

Figura 1. Total de los costo directos anuales de pacientes ambulatorios con EPOC con más de un año de diagnóstico por nivel de gravedad en dólares, 2014.

\section{Costos indirectos}

En los costos indirectos del paciente, sus familiares y la suma de ambos costos no fueron estadísticamente significativos de acuerdo con la gravedad de la EPOC (tabla 2).

\section{Costos sociales}

La suma de los costos directos e indirectos representa el costo social. El costo social anual fluctuó entre US $\$ 1,169$ de la categoría leve y US \$2,306 de la categoría muy grave. Los costos directos representaron entre 95.5 y $97.4 \%$ del costo social (tabla 2 ).

\section{DISCUSIÓN}

Es de nuestro conocimiento que éste es el primer estudio que describe la carga económica de la EPOC desde la perspectiva del paciente en México, ya que los estudios previos que conocemos la han descrito desde la perspectiva del sistema de salud, ${ }^{23,24}$ pero la descripción del gasto (costos directos e indirectos) realizado por el paciente (de su bolsillo) era necesaria para describir por completo los costos asociados a la EPOC; lo antes mencionado permitirá cumplir y planear intervenciones de políticas públicas en salud y además, valorar el impacto de los diversos sistemas de aseguramiento en salud.

La gravedad de la EPOC fue un buen predictor de los costos médicos directos, principalmente de los medicamentos y el oxígeno; y de los no médicos como el costo en alojamiento. En un estudio previo, los me- 
Tabla 2. Costos directos, indirectos y sociales anuales de pacientes ambulatorios con EPOC con más de un año de diagnóstico por nivel de gravedad en dólares, 2014. $(n=484)$.

\begin{tabular}{|c|c|c|c|c|c|}
\hline Costos & $\begin{array}{c}\text { Leve (I) } \\
\text { Mediana (p25, p75) }\end{array}$ & $\begin{array}{c}\text { Moderado (II) } \\
\text { Mediana (p25, p75) }\end{array}$ & $\begin{array}{c}\text { Grave (III) } \\
\text { Mediana (p25, p75) }\end{array}$ & $\begin{array}{c}\text { Muy grave (IV) } \\
\text { Mediana (p25, p75) }\end{array}$ & Valor $\mathrm{p}$ \\
\hline \multicolumn{6}{|l|}{ Costos directos } \\
\hline Consultas médicas & $11(7-14)$ & $14(7-21)$ & $18(12-25)$ & $18(9-36)$ & 0.0022 \\
\hline Pruebas clínicas & $5(3-21)$ & $12(5-37)$ & $20(9-42)$ & $12(5-51)$ & 0.0899 \\
\hline Medicamentos & 1,047 (909-1,102) & $1,182(1,113-1,261)$ & $1,302(1,236-1,411)$ & $1,460(1,366-1,539)$ & 0.0001 \\
\hline Oxígeno & $422(135-723)$ & $542(359-792)$ & $495(340-932)$ & $701(388-1057)$ & 0.0174 \\
\hline Equipo para desplazarse & & $44(34-63)$ & $4(4-18)$ & $15(3-35)$ & 0.0649 \\
\hline Transporte & $6(2-16)$ & $13(5-32)$ & $15(5-45)$ & $15(7-32)$ & 0.0468 \\
\hline Comida & $8(4-8)$ & $8(4-16)$ & $9(4-16)$ & $9(5-18)$ & 0.5479 \\
\hline Alojamiento & $106(106-106)$ & $91(30-220)$ & $106(45-121)$ & $106(45-212)$ & 0.9665 \\
\hline Total costos directos & $1,116(934-1,426)$ & $1,533(1,247-1,921)$ & $1,825(1,451-2,258)$ & $2,245(1,806-2,614)$ & 0.0001 \\
\hline \multicolumn{6}{|l|}{ Costos indirectos } \\
\hline Paciente & $141(141-141)$ & $56(15-84)$ & $38(21-56)$ & $55(26-138)$ & 0.2585 \\
\hline Familiares & $38(38-105)$ & 57 (33-92) & $55(20-76)$ & $42(25-102)$ & 0.5996 \\
\hline Total costos indirectos & $47(38-114)$ & $76(38-113)$ & $55(26-98)$ & $55(28-114)$ & 0.4652 \\
\hline Costo social & $1,169(943-1,474)$ & $1,605(1,306-2,031)$ & $1,887(1,528-2,343)$ & $2,306(1,863-2,758)$ & 0.0001 \\
\hline
\end{tabular}

dicamentos representaron uno de los costos directos médicos más cuantiosos y denotaron una correlación positiva con la gravedad de la enfermedad, ${ }^{11}$ como también lo mostraron nuestros resultados. La terapia de oxígeno también se ha descrito como una fuente importante de gasto para el paciente..$^{8-10}$ Nuestros resultados mostraron que incluso pacientes en las categorías de leve (I) y moderado (II) de la clasificación GOLD requirieron terapia de oxígeno, lo anterior se puede explicar por la altitud de la Ciudad de México que se encuentra a 2,240 m sobre el nivel del mar y empeora la hipoxemia haciendo mucho más probable la necesidad de oxígeno que en pacientes similares que residen a nivel del mar. Las consultas médicas y las pruebas clínicas representaron un bajo porcentaje de los gastos de bolsillo que debe realizar el paciente con EPOC debido al subsidio que realiza la Secretaría de Salud a través del INER, lo que implica una fuente importante de ahorro para los pacientes. A pesar de que la seguridad social y el subsidio ofrecido por el sistema de salud reducen los gastos de bolsillo, no los reducen a cero. En México es común que personas que cuentan con seguridad social opten por servicios privados de salud o por públicos fuera de su sistema de aseguramiento en busca de una mejor atención, lo que hace que incurran en gastos propios mayores. Dos tercios de los pacientes encuestados reportaron contar con una cobertura total en servicios de salud a través de la seguridad social que ofrece el estado, que por otro lado no cubre la atención fuera de los servicios que ofrece su sistema de aseguramiento, mientras que el resto de los pacientes son elegibles para recibir un subsidio en los servicios de salud dependiendo de su ingreso.

El costo directo no médico con mayor gasto fue el de alojamiento, debido a que cerca de $40 \%$ de los pacientes no vive en la Ciudad de México. Aun cuando muchos pacientes no pagan propiamente alojamiento, pasan en promedio 14 días anuales en casa de un familiar para poder asistir a sus consultas médicas. Si tuvieran que pagar esos días de alojamiento, gastarían US \$212.24 anuales; lo que implica que la casa de familiares o amigos son otra fuente de ahorro. En otros estudios no se estiman los costos médicos indirectos; sin embargo, nuestros resultados muestran que es muy importante considerar los costos de acuerdo con el contexto de los pacientes.

Los costos indirectos representaron una baja proporción de los costos sociales (2.5-4.6\%). Nuestra población de estudio fue en promedio 6 a 8 años mayor que en estudios similares. ${ }^{4-11}$ Lo anterior se reflejó en una alta proporción de pacientes jubilados, sólo un estudio en Italia reportó una distribución porcentual entre los costos directos e indirectos similar a la descrita con nuestros datos. ${ }^{9}$

La principal limitación potencial del estudio deriva de que los costos fueron estimados a partir de un interrogatorio del año previo a la inclusión del estudio y están sujetos a la memoria del paciente y su familia. Sin embargo, se redujo esta posibilidad, ya que por medio de los expedientes clínicos se verificó el número de consultas médicas, pruebas clínicas, la prescripción de medicamentos y comorbilidades, asimismo, se realizaron llamadas telefónicas para confirmar los datos cuando fue necesario. 
Por otro lado, el estudio se desarrolló en un hospital de referencia con pacientes en general con padecimientos más graves y los resultados no aplicarían para aquéllos que acuden a instituciones de primer y segundo nivel de atención. Sin embargo, los pacientes están bien caracterizados y aun cuando los costos monetarios en salud pueden variar en los otros niveles de atención, la tendencia de mayores costos a mayor gravedad está propiamente analizada. Con los costos estimados en este estudio se podría calcular con bastante aproximación la carga económica de la EPOC de acuerdo con la gravedad de la enfermedad, por lo menos en pacientes atendidos en instituciones de tercer nivel, ya que en el nivel primario muchos pacientes con EPOC no están diagnosticados (90\%) y por lo mismo no reciben los tratamientos recomendados, pero sí reciben medicamentos aunque no los ideales.

\section{CONCLUSIÓN}

Los costos directos se incrementan con la gravedad de la EPOC. Los principales gastos del bolsillo de los pacientes fueron los medicamentos, la terapia de oxígeno y el pago en alojamiento. Los costos directos representaron la proporción más grande de los costos sociales.

\section{Agradecimientos}

Agradecemos al personal médico del Departamento de Investigación de Tabaquismo y EPOC por su invaluable apoyo: Mónica Velázquez Uncal, Oliver Pérez Bautista, Candelaria Sánchez Romero, Alejandra Velázquez Montero, Leticia Ochoa Coutiño, Rafael Hernández, Luis Fernando Flores Trujillo y Carlos Espinosa de los Montero.

\section{REFERENCIAS}

1. World Health Organization (WHO). Chronic obstructive pulmonary disease (COPD). Fact sheet No 315. Access date: March 17, 2015. Available from: http://www.who. int/mediacentre/factsheets/fs315/en/

2. Ladeira I, Gomes T, Castro A, Ribeiro C, Guimarães M, Taveira N. The overall impact of COPD (CAT) and $B O D E$ index on COPD male patients: correlation? Rev Port Pneumol 2015;21(1):11-15. doi: 10.1016/j. rppnen.2014.02.004.

3. Aït-Khaled N, Enarson DA, Ottmani S, El Sony A, Eltigani $\mathrm{M}$, Sepulveda R. Chronic airflow limitation in developing countries: burden and priorities. Int J Chron Obstruct Pulmon Dis 2007;2(2):141-150.

4. Halpern MT, Stanford RH, Borker R. The burden of COPD in the U.S.A.: results from the Confronting COPD survey. Respir Med 2003;97 Suppl C:S81-89.
5. Britton M. The burden of COPD in the U.K.: results from the Confronting COPD survey. Respir Med 2003;97 Suppl $\mathrm{C}:$ S71-79.

6. Izquierdo JL. The burden of COPD in Spain: results from the Confronting COPD survey. Respir Med 2003;97 Suppl C:S61-69.

7. Wouters EF. The burden of COPD in The Netherlands: results from the Confronting COPD survey. Respir Med 2003;97 Suppl C:S51-59.

8. Piperno D, Huchon G, Pribil C, Boucot I, Similowski T. The burden of COPD in France: results from the Confronting COPD survey. Respir Med 2003;97 Suppl C:S33-42.

9. Dal Negro R, Rossi A, Cerveri I. The burden of COPD in Italy: results from the Confronting COPD survey. Respir Med 2003;97 Suppl C:S43-50.

10. Chapman KR, Bourbeau J, Rance L. The burden of COPD in Canada: results from the Confronting COPD survey. Respir Med 2003;97 Suppl C:S23-31.

11. Jansson SA, Andersson F, Borg S, Ericsson A, Jönsson $\mathrm{E}$, Lundbäck B. Costs of COPD in Sweden according to disease severity. Chest 2002;122(6):1994-2002.

12. Miller JD, Foster $T$, Boulanger $L$, et al. Direct costs of COPD in the U.S.: an analysis of Medical Expenditure Panel Survey (MEPS) data. COPD 2005;2(3):311-318.

13. Pérez N, Murillo R, Pinzón C, Hernández G. Costos de la atención médica del cáncer de pulmón, la EPOC y el IAM atribuibles al consumo de tabaco en Colombia (proyecto multicéntrico de la OPS). Rev Colomb Cancerol 2007;11(4):241-249.

14. Simoni-Wastila L, Blanchette CM, Qian J, et al. Burden of chronic obstructive pulmonary disease in Medicare beneficiaries residing in long-term care facilities. Am J Geriatr Pharmacother 2009;7(5):262-270. doi: 10.1016/j. amjopharm.2009.11.003.

15. Sharafkhaneh A, Petersen NJ, Yu HJ, Dalal AA, Johnson $\mathrm{ML}$, Hanania NA. Burden of COPD in a government health care system: a retrospective observational study using data from the US Veterans Affairs population. Int J Chron Obstruct Pulmon Dis 2010;5:125-132.

16. Dalal AA, Shah M, D'Souza AO, Rane P. Costs of inpatient and emergency department care for chronic obstructive pulmonary disease in an elderly Medicare population. J Med Econ 2010;13(4):591-598. doi: 10.3111/13696998.2010.521734.

17. Dalal AA, Christensen L, Liu F, Riedel AA. Direct costs of chronic obstructive pulmonary disease among managed care patients. Int J Chron Obstruct Pulmon Dis 2010;5:341-349. doi: 10.2147/COPD.S13771.

18. Masa JF, Sobradillo V, Villasante C, et al. Costes de la EPOC en España. Estimación a partir de un estudio epidemiológico poblacional. Arch Bronconeumol 2004;40(2):72-79.

19. Strâmbu I, Bucşa S, Nicolescu D, Dumitrescu C, Grigorescu B, Stoicescu IP. Cost analysis in patients admitted with COPD in 2002. Pneumologia 2004;53(4):161168.

20. Bakerly ND, Davies C, Dyer M, Dhillon P. Cost analysis of an integrated care model in the management of acute exacerbations of chronic obstructive pulmonary 
disease. Chron Respir Dis 2009;6(4):201-208. doi: $10.1177 / 1479972309104279$.

21. Menezes AM, Pérez-Padilla R, Jardim JR, et al. Chronic obstructive pulmonary disease in five Latin American cities (the PLATINO study): a prevalence study. Lancet 2005;366(9500):1875-1881.

22. Consenso Mexicano de EPOC; Ramírez VA, Sansores MR. Panorama epidemiológico e impacto económico actual. Neumol Cir Torax 2007;66(Supl 2):S13-S16.

23. Reynales-Shigematsu LM, Rodríguez-Bolaños RA, Jiménez JA, Juárez-Márquez SA, Castro-Ríos A, HernándezÁvila M. Costos de la atención médica atribuibles al consumo de tabaco en el Instituto Mexicano del Seguro Social. Salud Pública Mex 2006;48(Supl 1):S48-S64.

24. Reynales-Shigematsu LM, Juárez-Márquez SA, ValdésSalgado R. Costos en atención médica atribuibles al tabaquismo en el IMSS, Morelos. Salud Pública Mex 2005;47(6):451-457.

25. Cano-Valle F. Día Mundial de la EPOC. Rev Inst Nal Enf Resp Mex 2006;19(4):247.

26. Liljas B. How to calculate indirect costs in economic evaluations. Pharmacoeconomics 1998;13(1 Pt 1):1-7.

27. Comisión Nacional de Salarios Mínimos (CONASAMI). Tabla de salaries mínimos por área geográfica. Fecha de consulta: 14 de abril, 2015. Accesible en: http://www. conasami.gob.mx/t_sal_mini_prof.html.
28. World Bank (WB). GDP per capita (current US\$). Access date: April 14, 2015. Available from: http://data.worldbank.org/indicator/NY.GDP.PCAP.CD.

29. Organization for Economic Co-operation and Development (OCDE). Income nequality and Poverty. Figures and Data. Access date: April 14, 2015. Available from: http:// www.oecd.org/social/inequality-and-poverty.htm.

30. Herdman M, Badia X, Berra S. El EuroQol-5D: una alternativa sencilla para la medición de la calidad de vida relacionada con la salud en atención primaria. Aten Primaria 2001;28(6):425-429.

\section{$\triangle$ Correspondencia:}

M. en C. David Martínez Briseño

Instituto Nacional de Enfermedades Respiratorias

Ismael Cosío Villegas, Ciudad de México.

Calzada de Tlalpan Núm. 4502,

Colonia Sección XVI, C.P. 14080, Ciudad de México.

Teléfono: 5487-1700, extensión 5048,

fax 56-65-46-23

Correo electrónico: mb3david@gmail.com

Los autores declaran no tener conflicto de intereses. 